\title{
Comparison of deep underground neutron flux measured with a helium-3 filled proportional counter and evaluated from element composition or isotopic ratio of ${ }^{36} \mathrm{Cl} / \mathrm{Cl}$ in granite rock
}

\author{
By T. Kubota*, T. Ohta, Y. Mahara and A. Kudo \\ Kyoto University Research Reactor Institute, Kumatori, Sennan, Osaka 590-0494, Japan
}

(Received November 28, 2009; accepted in revised form December 20, 2010)

Neutron flux / Underground / $(\alpha, n)$ reaction / Spontaneous fission / AMS / Cl-36

\begin{abstract}
Summary. The chlorine isotopic ratio and elemental composition of two granite cores collected in a deep underground tunnel has been measured; neutrons were detected using a helium-3-filled proportional counter placed in a borehole. The thermal neutron flux determined from the chlorine isotopic ratio from accelerator mass spectrometry is $(3.8 \pm 0.2) \times$ $10^{-5}$ and that determined from the granite's elemental composition is $(4.4 \pm 0.3) \times 10^{-5}\left(\mathrm{~cm}^{-2} \mathrm{~s}^{-1}\right)$. The results of in-situ measurements using the proportional counter yielded a thermal neutron flux value of $(3.3 \pm 0.1) \times 10^{-5}\left(\mathrm{~cm}^{-2} \mathrm{~s}^{-1}\right)$. This good agreement among the results shows the validity of the neutron flux evaluation obtained from the elemental composition and/or the isotopic ratio of ${ }^{36} \mathrm{Cl} / \mathrm{Cl}$. Further, it reveals that the neutron flux in the underground environment remains stable for at least one million years, i.e., the period over which chlorine-36 attains equilibrium.
\end{abstract}

\section{Introduction}

A neutron generated underground is moderated to a thermal neutron, and this in turn is captured to produce a new nuclide. Thermal neutrons, as well as epithermal and fast neutrons, can be measured using a proportional counter filled with $\mathrm{He}-3$ or $\mathrm{BF}_{3}$ gas and by using some moderator if necessary [1-5]. The isotopic ratio of some nuclides with long half-life provides the value of thermal neutron flux under the condition that nuclide production is in equilibrium through a simple reaction with steady irradiation [6-8].

In a deep underground environment, neutrons are mostly generated through the spontaneous fission of uranium and through an $(\alpha, n)$ reaction between light elements and alpha particles from U- and Th-series nuclides; the presence of cosmogenic neutrons can be considered to be negligible. Neutron generation from elemental sources has been discussed in several reports [6,9-13]; however, little attention has been given to the role of neutron moderation [14].

In this study, the JENDL-3.3 neutron cross section data library is used to obtain a quantitative estimation of neu-

\footnotetext{
*Author for correspondence (E-mail: t_kubota@ rri.kyoto-u.ac.jp).
}

tron moderation. We have carried out a comparison among three methods for the determination of the thermal neutron flux; i.e., determination from element composition analysis, Cl-36 isotopic ratios, and in-situ measurements. If the estimation of neutron fluxes from the elemental composition was more quantitative, then the origin of neutrons and a fraction of cosmogenic neutrons in a shallow underground environment could be evaluated.

\section{Experimental}

Core sampling and neutron detection were conducted from November 2004 to January 2005 in a tunnel located in mountains in central Japan. The sampling point was surrounded by approximately 600 -m-thick granitic rock; therefore, the effect of cosmic rays on the measurements could be considered negligible. This long-term in-situ measurement was undisturbed due to constant temperature, humidity, and air current in the tunnel; further, public access to this area was restricted.

In this sampling period, two cores (diameters: $77 \mathrm{~mm}$ $(\varnothing 77)$ and $128 \mathrm{~mm}(\varnothing 128)$, respectively; length: $1500 \mathrm{~mm})$ were collected from the tunnel wall. The cores were fragmented during core collection. Based on the fragments, we obtained ten fractions $(1.0-5.0 \mathrm{~kg})$ from each core. Neutrons in one borehole $(\varnothing 77)$ were measured using a helium3-filled proportional counter (diameter: $50 \mathrm{~mm}$; length: $450 \mathrm{~mm}$; 3-atm helium-3), whereas the other borehole was used for another study. In this study, both core samples were used for elemental composition analysis and chlorine isotopic analysis to illustrate geological homogeneity, although only the former borehole was used for neutron counting.

For elemental composition analysis, the surface of the fraction samples, whose weathered blocks were first removed, was shaved off and washed with diluted $\mathrm{HNO}_{3}$ and then with ultrapure water. The fraction samples were broken into smaller chips by hammering and then milled into a fine powder. These powder samples were added to ultrapure water with ultrasonic agitation and then centrifuged to discard the supernatant. The latter treatment was conducted a total of three times. Finally, the powder samples were dried and then subjected to elemental composition analysis. The 
Table 1. Summary of element analysis.

\begin{tabular}{|c|c|}
\hline Element & Procedure \\
\hline $\begin{array}{l}\mathrm{Si}, \mathrm{Ti}, \mathrm{Al}, \mathrm{Fe}, \mathrm{Mn}, \\
\mathrm{Mg}, \mathrm{Ca}, \mathrm{Na}, \mathrm{K}, \mathrm{S}, \\
\mathrm{Cl}, \mathrm{Zr}, \mathrm{Ga}, \mathrm{Co}, \mathrm{O}^{(*)}\end{array}$ & $\mathrm{X}$-ray fluorescence analysis (XRF) \\
\hline B & Alkali fusion + ICP-AES \\
\hline $\mathrm{Sr}, \mathrm{Ba}, \mathrm{As}, \mathrm{P}$ & Acid treatment + ICP-AES \\
\hline $\begin{array}{l}\mathrm{Li}, \mathrm{Be}, \mathrm{Cr}, \mathrm{V}, \mathrm{Ni} \\
\mathrm{Cu}, \mathrm{Zr}, \mathrm{Rb}, \mathrm{Y}, \mathrm{Nb} \\
\mathrm{Pb}, \mathrm{Cd}, \mathrm{Sm}, \mathrm{Eu}, \mathrm{Gd} \\
\mathrm{Dy}, \mathrm{Yb}, \mathrm{Lu}, \mathrm{Th}, \mathrm{U}\end{array}$ & Acid treatment + ICP-MS \\
\hline $\mathrm{C}$ & Total organic carbon (TOC) analyzer \\
\hline Porosity & Gravimetric method \\
\hline \multicolumn{2}{|c|}{$\begin{array}{l}\text { Acid treatment: The powder sample in a Teflon beaker was added to } \\
\text { a mixture of } \mathrm{HNO}_{3}, \mathrm{HClO}_{4} \text {, and } \mathrm{HF} \text {; this was followed by heating to } \\
\text { completely dissolve rocks, and the resulting solution was diluted to } \\
100 \mathrm{ml} \text {. Alkali fusion: The powder sample and } \mathrm{Na}_{2} \mathrm{CO}_{3} \text { in a Pt crucible } \\
\text { were heated using a burner; the fused material, once cooled down, was } \\
\text { dissolved with hot dilute } \mathrm{HCl} \text {, followed by filtration. The filtrate was } \\
\text { diluted to } 100 \mathrm{ml} \text {. The concentration of oxygen was evaluated assuming } \\
\text { that these metals are present as oxides. }\end{array}$} \\
\hline
\end{tabular}

concentration of the elements that were considered to have contributed to the generation and capture of neutrons was measured by the methods summarized in Table 1. In addition to the elemental composition analysis, the porosity of powder samples was measured instead of water content in the granite sample due to measurement difficulties; nevertheless, water content is naturally important to the evaluation of thermal neutron flux. The evaluation in this study was conducted with the consideration of a reasonable range of water content in granite.

The chlorine isotopic ratio was measured by accelerator mass spectrometry (ETH AMS), for which an $\mathrm{AgCl}$ sample of several milligrams is required. The preparation of $\mathrm{AgCl}$ from powder samples was performed as follows: The powder sample was treated with a mixture of $\mathrm{HNO}_{3}$ and $\mathrm{HF}$ at $80^{\circ} \mathrm{C}$ for $48 \mathrm{~h}$. The resulting supernatant was transferred to a polypropylene centrifugation tube. The residual was washed twice with ultrapure water. The rinse solution was mixed in the centrifugation tube, and $\mathrm{AgNO}_{3}$ was added to precipitate $\mathrm{AgCl}$. After centrifugation, the supernatant was discarded. The precipitate was washed with ultrapure water and dissolved in $\mathrm{NH}_{4} \mathrm{OH}$, after which $\mathrm{Ba}\left(\mathrm{NO}_{3}\right)_{2}$ was added to precipitate $\mathrm{BaSO}_{4}$ for the removal of sulfur, which is harmful to AMS measurements. The discarding of the precipitate and the addition of $\mathrm{Ba}\left(\mathrm{NO}_{3}\right)_{2}$ was repeated while $\mathrm{BaSO}_{4}$ was still being precipitated. The resulting solution was finally added to $\mathrm{HNO}_{3}$ and $\mathrm{AgNO}_{3}$ to prepare the $\mathrm{AgCl}$ precipitate.

In-situ neutron measurement was performed by using a He-3-filled proportional counter with and without a cadmium shield (a 2-mm-thick cadmium sheet), which provided different responses to thermal and fast neutrons. A count for $50000 \mathrm{~s}$ at each measurement was repeated a total of 12 times to observe the temporal change in the neutron flux in a deep underground environment. The measurement appara- tus was covered with a polyethylene bag, and commercial power supply in the long tunnel was stabilized by using an uninterruptible power supply (UPS) to reduce the electrical noise.

\section{Estimation of thermal neutron flux}

The fate of neutrons generated through the spontaneous fission of uranium and an $(\alpha, n)$ reaction was evaluated as described below. Neutron generation from spontaneous fission is calculated from the fission half-life and the number of prompt neutrons per fission, which is 0.421 $\left(\mathrm{y}^{-1} \mathrm{~g}^{-1} \mathrm{ppm}^{-1}\right)$ [12]. This value is multiplied by the rock density and uranium concentration to obtain the neutron generation density $\left(\mathrm{cm}^{-3} \mathrm{~s}^{-1}\right)$.

Neutron production via an $(\alpha, n)$ reaction has been discussed in [9-13]. In these studies, the neutron yield was correlated with the values of the mass stopping power of alpha particles under a radioactive equilibrium of uranium and thorium series. The total alpha-induced neutron yield can be calculated, assuming that a sample is homogeneous, with the equation for neutron yield, which is described in [9] as

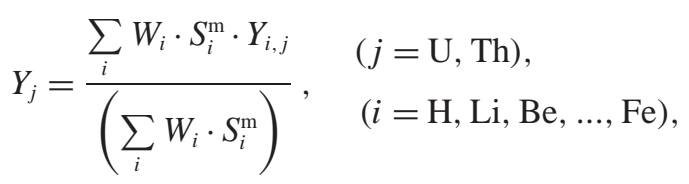

where $Y_{j}$ is the neutron yield related to alpha particles emitted from element $j$ and its daughter, $i$ denotes light elements, $W$ is the weight ratio of element $i$ to the sample compound, $S^{\mathrm{m}}$ is the mass stopping power, and $Y_{i, j}$ is the neutron yield for the thick target of element $i$. The two latter constants used are values that are summarized in [12].

Neutrons that are generated generally have high energy ranging from $0.1 \mathrm{MeV}$ to $10 \mathrm{MeV}$ [4], and they are moderated to a lower energy by underground material. The conversion ratio of neutrons generated to those that are moderated can be evaluated by the equation of the resonance escape probability, which takes into account the fact that some fraction of the generated neutrons is captured during their moderation; it is expressed as

$$
p\left(E_{\mathrm{a}} \rightarrow E_{\mathrm{b}}\right)=\exp \left[-\frac{1}{\xi} \int_{E_{\mathrm{b}}}^{E_{\mathrm{a}}} \frac{\Sigma_{\mathrm{a}}(E)}{\Sigma_{\mathrm{s}}+\Sigma_{\mathrm{a}}(E)} \frac{\mathrm{d} E}{E}\right]
$$

where $p\left(E_{\mathrm{a}} \rightarrow E_{\mathrm{b}}\right)$ is the resonance escape probability, i.e., the ratio of the number of surviving and moderated neutrons with energy $E_{\mathrm{b}}$ to the number of initially generated neutrons with energy $E_{\mathrm{a}} ; \xi$ is the mean lethargy gain per collision; and $\Sigma_{\mathrm{a}}$ and $\Sigma_{\mathrm{s}}$ are the macroscopic capture and scattering cross section, respectively, where $\Sigma_{\mathrm{s}}$ can be considered to be independent of energy.

The above equation is valid for an energy range from $1 \mathrm{eV}$ to $0.1 \mathrm{MeV}$; hence, in this evaluation, an energy of less than $1 \mathrm{eV}$ is considered as thermal energy. However, in the highenergy range $(0.1-10 \mathrm{MeV})$, the small neutron capture cross section and lack of significant resonance capture results in a slowing down density at $0.1 \mathrm{MeV}$ of unity; in other words, all neutrons are regarded as being generated with an energy of $0.1 \mathrm{MeV}$. 
When a neutron capture cross section obeys the 1/v law, $\Sigma_{\mathrm{a}}(E)$ can be expressed as

$$
\Sigma_{\mathrm{a}}(E)=\Sigma_{\mathrm{a}, \mathrm{th}} \sqrt{\frac{0.0253}{E}}
$$

where $\Sigma_{\text {ath }}$ is the thermal capture cross section. In such a case, the equation of the resonance escape probability is transformed as

$$
p\left(E_{\mathrm{a}} \rightarrow E_{\mathrm{b}}\right)=\exp \left[-2 \sqrt{0.0253} \frac{\Sigma_{\mathrm{a}, \mathrm{h}}}{\xi \Sigma_{\mathrm{s}}}\left(\frac{1}{\sqrt{E_{\mathrm{b}}}}-\frac{1}{\sqrt{E_{\mathrm{a}}}}\right)\right]
$$

When $\Sigma_{\mathrm{a}}(E)$ is constant, the equation can be transformed as

$$
p\left(E_{\mathrm{a}} \rightarrow E_{\mathrm{b}}\right)=\exp \left[-\frac{\Sigma_{\mathrm{a}}}{\xi \Sigma_{\mathrm{s}}} \ln \frac{E_{\mathrm{b}}}{E_{\mathrm{a}}}\right]
$$

The cross section and lethargy gain of compounds are expressed as

$$
\begin{aligned}
& \Sigma_{\mathrm{s}}=\sum_{i} \Sigma_{\mathrm{s}, \mathrm{i}} \\
& \Sigma_{\mathrm{a}}=\sum_{i} \Sigma_{\mathrm{a}, \mathrm{i}} \\
& \xi=\frac{\sum_{i} \xi_{\mathrm{i}} \Sigma_{\mathrm{s}, \mathrm{i}}}{\sum_{i} \Sigma_{\mathrm{s}, \mathrm{i}}}
\end{aligned}
$$

where $\Sigma_{\mathrm{s}, \mathrm{i}}, \Sigma_{\mathrm{a}, \mathrm{i}}$, and $\xi_{\mathrm{i}}$ are the scattering cross section, capture cross section, and lethargy gain of component element $i$ in the compound, respectively.

The thermal neutron production density, which is the product of the neutron generation density $(S)$ and resonance escape probability $(p)$, is equal to the thermal neutron capture density, which is the product of the thermal neutron capture macroscopic cross section and the thermal neutron flux $\left(\varphi_{\text {th }}\right)$, because the number of neutrons is balanced between production and capture. This relationship shows that the thermal neutron flux can be evaluated using the following equation:

$$
\varphi_{\mathrm{th}}=\frac{1}{\Sigma_{\mathrm{a}}} p S
$$

where $\Sigma_{\mathrm{a}}, S$, and $p$ are determined from the results of the element analysis, which in turn provides the thermal neutron flux.

Deep underground, $\mathrm{Cl}-36$ is produced from $\mathrm{Cl}-35$ through neutron activation; because the flux of cosmic rays is completely shielded, the production of $\mathrm{Cl}-36$ from the spallation of calcium and potassium is negligible. The saturation activity of $\mathrm{Cl}-36$ by a constant neutron flux is given by

$$
A_{36}=\lambda_{36} N_{36}=N_{35} \sigma_{\mathrm{th}} \varphi_{\mathrm{th}}
$$

where $A_{36}$ is the saturation activity of Cl-36; $\lambda_{36}$ is its decay constant $\left(7.30 \times 10^{-14} \mathrm{~s}^{-1}\right) ; N_{36}$ and $N_{35}$ are the number of Cl-36 and Cl-35 atoms, respectively; and $\sigma_{\text {th }}$ is the thermal neutron capture cross section of Cl-35 (43.6 b). The production of $\mathrm{Cl}-36$ by fast neutrons is negligible because of the small cross section and low flux of fast neutrons. The thermal neutron flux calculated from the chlorine isotopic ratio is expressed as

$$
\varphi_{\text {th }}=\frac{\lambda_{36}}{\sigma_{\text {th }}} \cdot \frac{N_{36}}{N_{35}}=1.67 \times 10^{9} \cdot \frac{N_{36}}{N_{35}} .
$$

Although a He-3-filled proportional counter is highly sensitive to thermal neutrons, even fast neutrons are detected to a slight degree, and therefore, the contribution of fast neutrons should be subtracted from the measurements. For the simple evaluation of thermal neutron flux, we consider that all thermal neutrons were captured by the cadmium shield and that all fast neutrons passed through it. The counting rate without a cadmium shield $\left(C_{\text {Bare }}\right)$ and with a cadmium shield $\left(C_{C d}\right)$ is expressed as

$$
C_{\mathrm{Bare}}=\varepsilon_{\mathrm{th}} \cdot \varphi_{\mathrm{th}}+\varepsilon_{\mathrm{f}} \cdot \varphi_{\mathrm{f}}
$$

and

$$
C_{\mathrm{Cd}}=\varepsilon_{\mathrm{f}} \cdot \varphi_{\mathrm{f}},
$$

respectively, where the subscripts th and $\mathrm{f}$ indicate thermal and fast neutron flux, respectively, and $\varepsilon$ is the conversion factor between the counting rate and the neutron flux. The value of $\varepsilon_{\text {th }}$ that is used is indicated on the proportional counter as $100\left(\mathrm{cps} / \mathrm{cm}^{-2} \mathrm{~s}^{-1}\right)$. From the above two equations, after subtracting the contribution of fast neutrons, the thermal neutron flux is expressed as

$$
\varphi_{\mathrm{th}}=\frac{C_{\mathrm{Bare}}-C_{\mathrm{Cd}}}{\varepsilon_{\mathrm{th}}}=\frac{C_{\mathrm{Bare}}-C_{\mathrm{Cd}}}{100} .
$$

\section{Results and discussion}

The elemental composition analysis shows that seven major elements account for more than $99 \%$ of the components. Uranium and thorium, key elements in neutron production, account for approximately 2 and $10 \mathrm{ppm}$, respectively, and lanthanides with a large neutron capture cross section account for approximately $10 \mathrm{ppm}$; the geological homogeneity of the sampling location results in only a small variation in the elemental concentrations in each fraction (Table 2).

By using the average of ten fractions in each core as a representative value and Eq. (1), we can obtain the neutron production density as

$$
S=\rho(1.41[\mathrm{U}]+0.47[\mathrm{Th}]) \cdot \frac{1}{3.15 \times 10^{7}}
$$

where $\rho$ is the mass density of the core sample and [U] and [Th] are the element concentrations in ppm units.

The neutron flux was calculated from data from the JENDL-3.3 library. The neutron moderation, in particular, was calculated using 70-group cross section data. The neutron cross section of the core sample $(\varnothing 77)$ is shown in Fig. 1; the other core $(\varnothing 128)$ had a similar aspect. The macroscopic neutron capture cross section decreases according to the $1 / v$ law at energies less than $1 \mathrm{keV}$, and resonance capture, although small as compared to scattering, is significant at energies greater than $1 \mathrm{keV}$. The thermal neutron 
Table 2. Results of element analysis of all core sample fractions.

\begin{tabular}{|c|c|c|c|}
\hline & Aver & value & \\
\hline & Core sample ( $\varnothing 77)$ & Core sample $(\varnothing 128)$ & \\
\hline $\mathrm{O}$ & $49.5 \pm 0.1$ & $49.3 \pm 0.1$ & $\%$ \\
\hline $\mathrm{Si}$ & $34.2 \pm 0.2$ & $34.2 \pm 0.1$ & \\
\hline $\mathrm{Al}$ & $7.22 \pm 0.07$ & $7.29 \pm 0.06$ & \\
\hline $\mathrm{Fe}$ & $1.49 \pm 0.10$ & $1.50 \pm 0.04$ & \\
\hline $\mathrm{Ca}$ & $1.28 \pm 0.08$ & $1.29 \pm 0.05$ & \\
\hline $\mathrm{Na}$ & $2.35 \pm 0.06$ & $2.33 \pm 0.05$ & \\
\hline K & $4.06 \pm 0.13$ & $4.07 \pm 0.12$ & \\
\hline $\mathrm{Ti}$ & $0.13 \pm 0.01$ & $0.13 \pm 0.01$ & \\
\hline $\mathrm{Mg}$ & $0.28 \pm 0.03$ & $0.28 \pm 0.01$ & \\
\hline $\mathrm{Mn}$ & $314 \pm 31$ & $311 \pm 12$ & $\mathrm{ppm}$ \\
\hline $\mathrm{P}$ & $289 \pm 25$ & $324 \pm 20$ & \\
\hline S & $26.4 \pm 4.4$ & $31.4 \pm 13.7$ & \\
\hline $\mathrm{Cl}$ & $144 \pm 24$ & $143 \pm 10$ & \\
\hline V & $11.7 \pm 1.3$ & $13.6 \pm 1.0$ & \\
\hline $\mathrm{Cr}$ & $1.24 \pm 0.38$ & $1.25 \pm 0.26$ & \\
\hline Co & $47.4 \pm 4.9$ & $52.7 \pm 4.2$ & \\
\hline $\mathrm{Ni}$ & $1.17 \pm 0.47$ & $0.78 \pm 0.19$ & \\
\hline $\mathrm{Cu}$ & $2.93 \pm 0.28$ & $3.71 \pm 0.54$ & \\
\hline $\mathrm{Zn}$ & $21.8 \pm 2.3$ & $23.2 \pm 2.0$ & \\
\hline $\mathrm{Ga}$ & $12.7 \pm 1.1$ & $13.0 \pm 0.9$ & \\
\hline As & $0.85 \pm 0.48$ & $1.21 \pm 0.31$ & \\
\hline $\mathrm{Rb}$ & $123 \pm 3$ & $116 \pm 9$ & \\
\hline $\mathrm{Sr}$ & $133 \pm 28$ & $160 \pm 11$ & \\
\hline $\mathrm{Y}$ & $14.7 \pm 1.4$ & $14.1 \pm 1.8$ & \\
\hline $\mathrm{Zr}$ & $104 \pm 5$ & $105 \pm 4$ & \\
\hline $\mathrm{Nb}$ & $6.85 \pm 0.65$ & $7.18 \pm 0.76$ & \\
\hline $\mathrm{Ba}$ & $573 \pm 89$ & $712 \pm 81$ & \\
\hline $\mathrm{Pb}$ & $10.8 \pm 0.4$ & $10.7 \pm 0.3$ & \\
\hline $\mathrm{U}$ & $1.61 \pm 0.17$ & $1.51 \pm 0.19$ & \\
\hline Th & $11.5 \pm 1.6$ & $11.6 \pm 1.1$ & \\
\hline B & $0.65 \pm 0.22$ & $0.70 \pm 0.28$ & \\
\hline $\mathrm{Li}$ & $13.2 \pm 1.7$ & $12.7 \pm 0.4$ & \\
\hline $\mathrm{Be}$ & $2.01 \pm 0.47$ & $1.88 \pm 0.30$ & \\
\hline $\mathrm{Cd}$ & $0.03 \pm 0.01$ & $0.02 \pm 0.01$ & \\
\hline Gd & $3.95 \pm 0.64$ & $3.04 \pm 0.17$ & \\
\hline $\mathrm{Sm}$ & $3.56 \pm 0.42$ & $3.37 \pm 0.15$ & \\
\hline $\mathrm{Eu}$ & $0.82 \pm 0.11$ & $0.65 \pm 0.03$ & \\
\hline Dy & $2.18 \pm 0.12$ & $2.15 \pm 0.19$ & \\
\hline $\mathrm{Yb}$ & $1.41 \pm 0.09$ & $1.30 \pm 0.14$ & \\
\hline $\mathrm{Lu}$ & $0.19 \pm 0.01$ & $0.18 \pm 0.02$ & \\
\hline $\mathrm{F}$ & $194 \pm 21$ & $182 \pm 9$ & \\
\hline $\mathrm{C}$ & $54.2 \pm 22.5$ & $48.9 \pm 12.9$ & \\
\hline Porosity & $1.0 \pm 0.2$ & $0.9 \pm 0.1$ & $\%$ \\
\hline $\mathrm{H}_{2} \mathrm{O}^{*}$ & $0.01 \pm 0.01$ & $0.02 \pm 0.01$ & $\mathrm{~g} / \mathrm{cm}^{3}$ \\
\hline
\end{tabular}

The error value represents the standard deviation. The water content is evaluated by assuming that a pore in granite is saturated with water at one atm.

cross sections and lethargy gains are listed in Table 3, and the thermal neutron flux is calculated for the following three assumptions at energies greater than $1 \mathrm{keV}$ : (1) $\Sigma_{\mathrm{a}}(E)$ obeys only the $1 / v$ law and has no resonance capture, $(2) \Sigma_{\mathrm{a}}(E)$ is constant as $\Sigma_{\mathrm{a}}(1 \mathrm{keV})$, and (3) $\Sigma_{\mathrm{a}}(E)$ is ten times the original $\Sigma_{\mathrm{a}}(E)$. In all three assumptions, $\Sigma_{\mathrm{a}}(E)$ obeys the $1 / v$ law at energies less than $1 \mathrm{keV}$, and the three assumed $\Sigma_{\mathrm{a}}(E)$ are shown in Fig. 1. The results of each assumption are summarized in Table 3 and are in good agreement. The errors of assumption (1) derived from the maximum and minimum element concentration are listed in Table 2; the maximum concentration yields the lowest capture escape probability. The deviation of the macroscopic capture cross section has no effect upon neutron moderation. This is because the energy region capture cross section is approximately $1 / 1000$

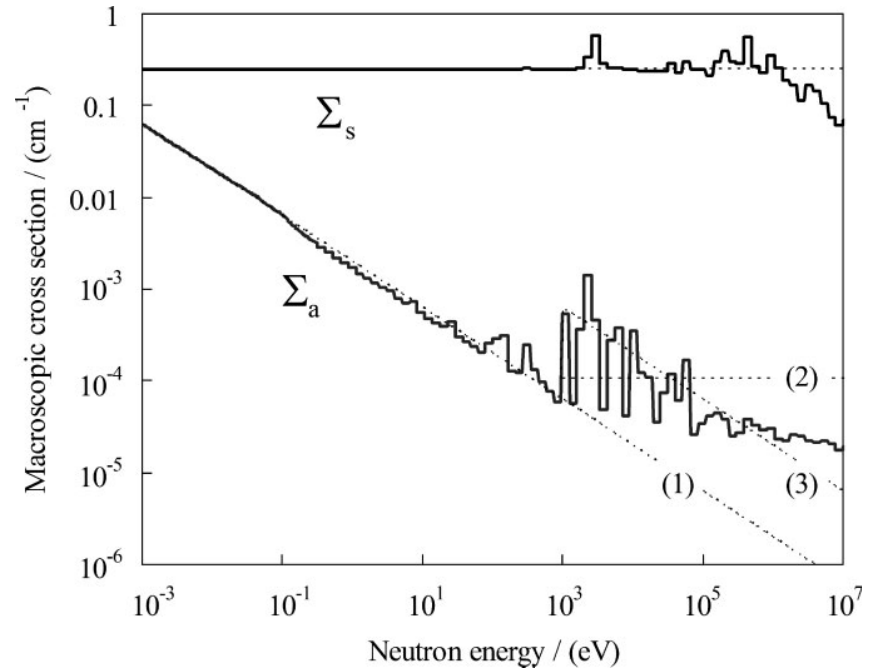

Fig. 1. Macroscopic cross section of the core sample ( $\varnothing 77)$. (Upper) macroscopic scattering cross section $\left(\Sigma_{\mathrm{s}}\right)$. (Lower) macroscopic capture cross section $\left(\Sigma_{\mathrm{a}}\right)$. The broken lines indicate cross sections used in the neutron moderation equation. The number denotes the following assumptions of capture cross section at neutron energies greater than $1 \mathrm{keV}$ : (1) $\Sigma_{\mathrm{a}}(E)$ obeys only the $1 / v$ law and has no resonance capture, (2) $\Sigma_{\mathrm{a}}(E)$ is constant as $\Sigma_{\mathrm{a}}(1 \mathrm{keV})$, (3) $\Sigma_{\mathrm{a}}(E)$ is ten times the original $\Sigma_{\mathrm{a}}(E)$. In all three assumptions, $\Sigma_{\mathrm{a}}(E)$ obeys the $1 / v$ law at energies less than $1 \mathrm{keV}$.

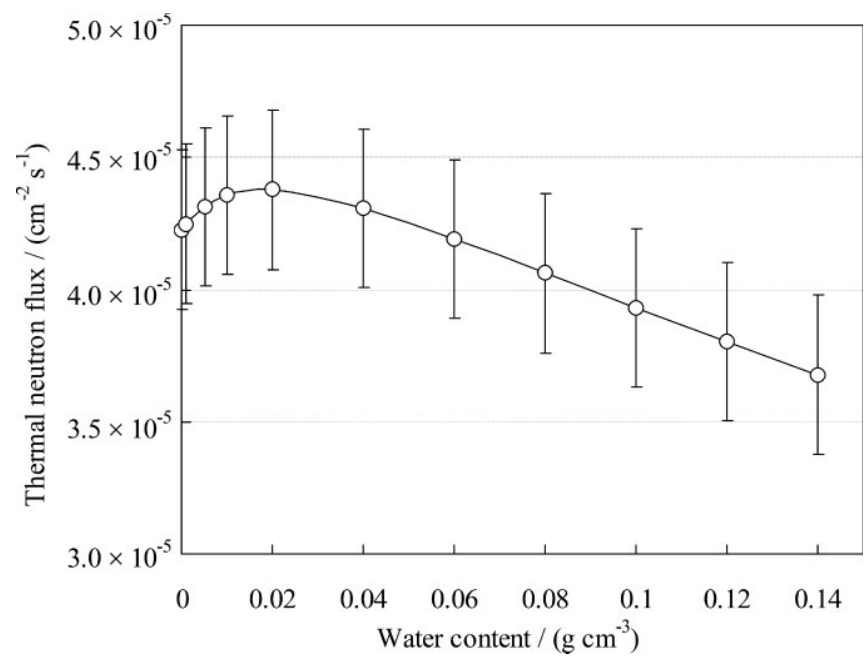

Fig. 2. The dependence on water content in granite for the evaluation of thermal neutron flux for core sample $(\varnothing 77)$. The error bars denoted originate from the error of the elemental composition analysis.

of the scattering cross section, and hence, the neutrons that interact with nuclides are almost completely scattered and lose their energy; only $0.1 \%$ of them end up being captured. The neutron generation density, resonance escape probability, and thermal neutron capture cross section can be used to determine the thermal neutron flux using Eqs. (15), (4), and (9), respectively. Finally, the element analysis of the two cores provides a thermal neutron flux of $(4.4 \pm 0.3) \times$ $10^{-5}\left(\mathrm{~cm}^{-2} \mathrm{~s}^{-1}\right)$.

The thermal neutron flux was evaluated by assuming a water content of $0.01 \mathrm{~g} / \mathrm{cm}^{3}$ in granite. The validity of this assumption is illustrated in Fig. 2. The evaluation results show negligible dependence on water content from 0 to $0.06 \mathrm{~g} / \mathrm{cm}^{3}$; however, the thermal flux peaks at $0.02 \mathrm{~g} / \mathrm{cm}^{3}$. Because of the low degree of water content for compact 
Table 3. Summary of neutron generation and moderation and thermal neutron flux.

\begin{tabular}{lcc}
\hline Core sample series & $\varnothing 77$ & $\varnothing 128$ \\
\hline Average dry mass density of core sample $\left(\mathrm{g} \mathrm{cm}^{-3}\right)$ & $2.60 \pm 0.01$ & $2.60 \pm 0.01$ \\
Macroscopic scattering cross-section, $\Sigma_{\mathrm{s}}\left(\mathrm{cm}^{-1}\right)$ & $0.26 \pm 0.01$ & $0.28 \pm 0.01$ \\
Lethargy gain, $\xi$ & $0.15 \pm 0.03$ & $0.19 \pm 0.04$ \\
Neutron generation density, $S\left(\mathrm{~cm}^{-3} \mathrm{~s}^{-1}\right)$ & $(6.4 \pm 0.7) \times 10^{-7}$ & $(6.3 \pm 0.5) \times 10^{-7}$ \\
Resonance escape probability, $p$ & estimation $1^{*}$ & \\
& $0.90 \pm 0.02$ & $0.92 \pm 0.01$ \\
& estimation 2 & 0.92 \\
& $\begin{array}{l}0.90 \\
\end{array}$ & 0.88 \\
Thermal neutron production density, $p S\left(\mathrm{~cm}^{-3} \mathrm{~s}^{-1}\right)$ & $(5.7 \pm 0.6) \times 10^{-7}$ & $(5.8 \pm 0.5) \times 10^{-7}$ \\
Macroscopic thermal neutron capture cross-section & $(1.31 \pm 0.04) \times 10^{-2}$ & $(1.30 \pm 0.02) \times 10^{-2}$ \\
$\Sigma_{\text {a,th }}\left(\mathrm{cm}^{-1}\right)$ & $(4.4 \pm 0.5) \times 10^{-5}$ & $(4.5 \pm 0.4) \times 10^{-5}$ \\
Thermal neutron flux $\left(\mathrm{cm}^{-2} \mathrm{~s}^{-1}\right)$ & $(4.4 \pm 0.3) \times 10^{-5}$ & \\
Average thermal neutron flux $\left(\mathrm{cm}^{-2} \mathrm{~s}^{-1}\right)$ & & \\
\hline
\end{tabular}

(*) Estimation 1 provides the thermal neutron production density.

Table 4. Chlorine-36 isotopic ratio of sample and estimation of thermal neutron flux.

\begin{tabular}{lccc}
\hline Core fraction & $\begin{array}{c}{ }^{36} \mathrm{Cl} /{ }^{35} \mathrm{Cl} \\
\left(\times 10^{-14}\right)\end{array}$ & Core fraction & $\begin{array}{c}{ }^{36} \mathrm{Cl} /{ }^{35} \mathrm{Cl} \\
\left(\times 10^{-14}\right)\end{array}$ \\
\hline$\varnothing 77-1$ & $2.6 \pm 0.4$ & $\varnothing 128-1$ & $1.7 \pm 0.3$ \\
$\varnothing 77-2$ & $1.7 \pm 0.3$ & $\varnothing 128-2$ & $2.2 \pm 0.3$ \\
$\varnothing 77-3$ & $2.1 \pm 0.3$ & $\varnothing 128-3$ & $2.1 \pm 0.3$ \\
$\varnothing 77-4$ & $2.4 \pm 0.3$ & $\varnothing 128-4$ & $1.8 \pm 0.3$ \\
$\varnothing 77-5$ & $2.0 \pm 0.3$ & $\varnothing 128-5$ & $2.2 \pm 0.3$ \\
$\varnothing 77-6$ & $2.4 \pm 0.3$ & $\varnothing 128-6$ & $2.2 \pm 0.4$ \\
$\varnothing 77-7$ & $2.1 \pm 0.3$ & $\varnothing 128-7$ & $2.6 \pm 0.4$ \\
$\varnothing 77-8$ & $2.8 \pm 0.4$ & $\varnothing 128-8$ & $2.4 \pm 0.3$ \\
$\varnothing 77-9$ & $2.4 \pm 0.3$ & $\varnothing 128-9$ & $2.4 \pm 0.3$ \\
$\varnothing 77-10$ & $2.4 \pm 0.3$ & $\varnothing 128-10$ & $2.5 \pm 0.4$ \\
Each average & $2.3 \pm 0.1$ & & $2.2 \pm 0.1$ \\
Total average & $2.3 \pm 0.1$ & & \\
Thermal & $(3.8 \pm 0.2) \times 10^{-5}\left(\mathrm{~cm}^{-2} \mathrm{~s}^{-1}\right)$ & \\
neutron flux & & & \\
\hline
\end{tabular}

The error value represents the standard deviation.

granite, the thermal neutron flux presented in this paper is reasonable.

All 20 samples from the two cores had similar chlorine isotopic ratios and no appreciable isotopic drift (Table 4). The average isotopic ratio $\left({ }^{36} \mathrm{Cl} /{ }^{35} \mathrm{Cl}\right)$ of both cores was $(2.3 \pm 0.1) \times 10^{-14}$. Based on this value, we obtained a thermal neutron flux of $(3.8 \pm 0.2) \times 10^{-5}\left(\mathrm{~cm}^{-2} \mathrm{~s}^{-1}\right)$ under the assumption that $\mathrm{Cl}-36$ was in equilibrium.

Each measurement by the He-3-filled proportional counter, both with and without a cadmium shield, was in agreement, with deviations of $14 \%$ and $6.8 \%$, respectively. The deviations were derived from the low neutron flux (Table 5). The deep underground thermal and fast neutron flux, although low, was considered stable; as a result, the thermal neutron flux was evaluated from the total counts made during measurements for $600000 \mathrm{~s}$. The thermal neutron flux was estimated as $(4.32 \pm 0.08) \times 10^{-3} \mathrm{cps}$ for $C_{\text {Bare }}$ and $(1.03 \pm 0.04) \times 10^{-3} \mathrm{cps}$ for $C_{\mathrm{Cd}}$; this gives a value of $(3.3 \pm 0.1) \times 10^{-5}\left(\mathrm{~cm}^{-2} \mathrm{~s}^{-1}\right)$.

The values for thermal neutron fluxes in a deep underground environment obtained from elemental composition
Table 5. Results of in-situ measurement and estimation of thermal neutron flux.

\begin{tabular}{lccccc}
\hline $\begin{array}{l}\text { Count } \\
\text { No. }\end{array}$ & $\begin{array}{c}\text { Count } \\
(50000 \mathrm{~s})\end{array}$ & Shield & $\begin{array}{c}\text { Count } \\
\text { No. }\end{array}$ & $\begin{array}{c}\text { Count } \\
(50000 \mathrm{~s})\end{array}$ & Shield \\
\hline$\# 1$ & $237 \pm 15.4$ & Bare & $\# 13$ & $40 \pm 6.3$ & Cadmium \\
$\# 2$ & $240 \pm 15.5$ & & $\# 14$ & $64 \pm 8.0$ & \\
$\# 3$ & $227 \pm 15.1$ & & $\# 15$ & $41 \pm 6.4$ & \\
$\# 4$ & $220 \pm 14.8$ & & $\# 16$ & $48 \pm 6.9$ & \\
$\# 5$ & $200 \pm 14.1$ & & $\# 17$ & $41 \pm 6.4$ & \\
$\# 6$ & $199 \pm 14.1$ & & $\# 18$ & $51 \pm 7.1$ & $59 \pm 7.7$ \\
$\# 7$ & $223 \pm 14.9$ & & $\# 20$ & $45 \pm 6.7$ & \\
$\# 8$ & $195 \pm 14.0$ & & $\# 21$ & $49 \pm 7.0$ & \\
$\# 9$ & $203 \pm 14.2$ & & $\# 22$ & $64 \pm 8.0$ & \\
$\# 10$ & $194 \pm 13.9$ & & $\# 23$ & $53 \pm 7.3$ & \\
$\# 11$ & $225 \pm 15.0$ & & $\# 24$ & $62 \pm 7.9$ & \\
$\# 12$ & $228 \pm 15.1$ & & & \\
Counting & $(4.32 \pm 0.08) \times 10^{-3}$ & Counting & $(1.03 \pm 0.04) \times 10^{-3}$ \\
rate (cps) & & rate (cps) & & \\
\hline
\end{tabular}

Thermal neutron flux $(3.3 \pm 0.1) \times 10^{-5}\left(\mathrm{~cm}^{-2} \mathrm{~s}^{-1}\right)$

The error value is estimated from the statistical error.

analysis, ${ }^{36} \mathrm{Cl} / \mathrm{Cl}$ isotopic ratio, and in-situ measurement are in good agreement. This shows that the assumption that $\mathrm{Cl}-36$ is in equilibrium is valid, and that the geological formation has been stable for at least one million years. In addition, this agreement shows that the thermal neutron flux estimation obtained by elemental composition is also valid, and that this estimation is quite useful for determining thermal neutron flux deep underground where in-situ measurements are difficult.

Our in-situ measurements, however, show a slightly low neutron flux, which may be because the cadmium shielding was insufficient for thermal neutrons; thus, the counting rate increased, and consequently, the resulting thermal neutron flux was underestimated. By eliminating this problem, a thermal neutron flux of at most $4.5 \times 10^{-5}\left(\mathrm{~cm}^{-2} \mathrm{~s}^{-1}\right)$, as derived from $C_{\mathrm{Bare}}$, and greater than $3.3 \times 10^{-5}\left(\mathrm{~cm}^{-2} \mathrm{~s}^{-1}\right)$ is obtained, which is in better agreement with the estimation of the thermal neutron flux value from the element composition. 
In this study, we have investigated the methods for the estimation of thermal neutron flux described in [6], where neutron capture during moderation is ignored. We have taken this capture into account in order to obtain a thermal neutron flux from the element composition analysis that is in good agreement with the value obtained from other measurements.

\section{References}

1. Rindi, A., Celani, F., Lindozzi, M., Miozzi, S.: Underground neutron flux measurement. Nucl. Instrum. Methods Phys. Res. A 272, 871 (1988).

2. Braoudakis, G., Peak, L. S.: Investigation of the background gamma spectrum in an underground environment. Nucl. Instrum. Methods Phys. Res. A 332, 292 (1993).

3. Arpesella, C.: A low background counting facility at Laboratori Nazionali del Gran Sasso. Appl. Radiat. Isotopes 47, 991 (1996).

4. Chazal, V., Brissot, R., Cavaignac, J. F., Chambona, B., de Jesus, M., Drain, D., Giraud-Heraud, Y., Pastor, C., Stutz, A., Vagneron, L.: Neutron background measurements in the underground laboratory of Modane. Astropart. Phys. 9, 163 (1998).

5. Carmona, J. M., Cebrián, S., García, E., Irastorza, I. G., Luzón, G., Morales, A., Morales, J., Ortiz de Solórzano, A., Puimedón, J., Sarsa, M. L., Villar, J. A.: Neutron background at the Canfranc underground laboratory and its contribution to the IGEX-DM dark matter experiment. Astropart. Phys. 21, 523 (2004).
6. Andrews, J. N., Fontes, J.-Ch., Michelot, J.-L., Elmore, D.: In-situ neutron flux, ${ }^{36} \mathrm{C} 1$ production and groundwater evolution in crystalline rocks at Stripa, Sweden. Earth Planet. Sci. Lett. 77, 49 (1986).

7. Andrews, J. N., Florkowski, T., Lehmann, B. E., Loosl, H. H.: Underground production of radionuclides in the Milk River aquifer, Alberta, Canada. Appl. Geochem. 6, 425 (1991).

8. Balderer, W., Synal, H.-A.: Tracing groundwater from uranium rich environments by ${ }^{36} \mathrm{Cl}$ isotope method: possibilities for natural analogue studies. Nucl. Instrum. Methods Phys. Res. B 223/224, 471 (2004)

9. Feige, Y., Oltman, B. G., Kastner, J.: Production rates of neutrons in soils due to natural radioactivity. J. Geophys. Res. 73, 3135 (1968).

10. Kuhn, M. W., Davis, S. N., Bentley, H. W.: Measurements of thermal neutrons in the subsurface. Geophys. Res. Lett. 11, 607 (1984).

11. Andrews, J. N., Davis, S. N., Fabryka-Martin, J., Fontes, J.-Ch., Lehmann, B.E , Loosli, H. H. , Michelot, J.-L., Moser, H., Smith, B., Wolf, M.: The in situ production of radioisotopes in rock matrices with particular reference to the Stripa granite. Geochim. Cosmochim. Acta 53, 1803 (1989).

12. Heaton, R., Lee, H., Skensved, P., Robertson, B. C.: Alpha-induced neutron activity in materials. Nucl. Geophys. 4, 499 (1990).

13. Lehmann, B. E., Loosli, H. H., Rauber, D., Thonnard, N., Willis, R. D.: ${ }^{81} \mathrm{Kr}$ and ${ }^{85} \mathrm{Kr}$ in groundwater, Milk River aquifer, Alberta, Canada. Appl. Geochem. 6, 419 (1991).

14. Florkowski, T., Kostka, T., Kotas, M.: Measurement of underground neutron flux. Nucl. Geophys. 6, 243 (1992). 